\title{
COMPLEMENTARY COMPONENTS OF POLYNOMIAL HULLS ${ }^{1}$
}

\author{
RICHARD F. BASENER
}

\begin{abstract}
Let $X$ be a compact subset of the unit sphere $S$ in $\mathbf{C}^{n}, n>1$. It is shown that if a point $z$ is not in the polynomially convex hull of $X$, then there is a complementary component $U$ of $X$ in $S$ such that $z$ is not in the hull of $S \backslash U$.
\end{abstract}

Alexander [1] has demonstrated several consequences of Browder's Theorem that $H^{i}(X)=0$ when $X$ is a polynomially convex subset of $\mathbf{C}^{n}$ and $i \geqslant n$. One of these is that the polynomially convex hull of a compact subset of a sphere (in $\mathbf{C}^{n}, n \geqslant 2$ ) has the same number of complementary components inside the sphere as the set itself has on the sphere. Here we use this result to further elaborate the role of complementary components in determining the hull of such a set.

Fix $n \geqslant 2$ and let $S=\left\{z \in \mathbf{C}^{n}:|z|=\left(\Sigma\left|z_{j}\right|^{2}\right)^{1 / 2}=1\right\}, B=\left\{z \in \mathbf{C}^{n}:|z|<\right.$ 1). For a compact subset $X$ of $\mathbf{C}^{n}$, let $\hat{X}$ denote the polynomially convex hull of $X$. For $Y \subseteq \mathbf{C}^{n}, \bar{Y}$ is the closure of $Y$ and $\partial Y$ is the boundary of $Y$ relative to $\mathrm{C}^{n}$. If $Y \subseteq S, \partial_{S} Y$ will denote the boundary of $Y$ relative to $S$.

Our main result is:

THEOREM. If $U_{1}, \ldots, U_{N}(1 \leqslant N \leqslant \infty)$ are disjoint open subsets of $S$, then

$$
\left(S \backslash \cup U_{j}\right)^{\wedge}=\cap\left(S \backslash U_{j}\right)^{\wedge} \text {. }
$$

Thus in order to compute the hull of a subset $X$ of $S$, it is sufficient to be able to describe the hulls of subsets of $S$ with connected complements, for we can now write $\hat{X}=\cap\left(S \backslash U_{j}\right)$, taking $U_{1}, U_{2}, \ldots$ to be the components of $S \backslash X$. Before proceeding to the proof we mention some immediate consequences.

Corollary 1. Suppose that $\Delta_{1}, \ldots, \Delta_{N}(1 \leqslant N \leqslant \infty)$ are disjoint open "spherical caps", i.e., for each $j$ there is $a z_{j} \in S$ and an $\varepsilon_{j}, 0<\varepsilon_{j}<2$, with $\Delta_{j}=\left\{z \in S:\left|z-z_{j}\right|<\varepsilon_{j}\right\}$. Then

$$
\left(S \backslash \cup \Delta_{j}\right)^{\wedge}=\text { linear convex hull }\left(S \backslash \cup \Delta_{j}\right) \text {. }
$$

Proof. For each $j,\left(S \backslash \Delta_{j}\right)^{\wedge}=$ linear convex hull $\left(S \backslash \Delta_{j}\right)$.

Received by the editors July 14, 1977.

AMS (MOS) subject classifications (1970). Primary 32E20.

Key words and phrases. Polynomially convex hull, several complex variables.

'This research was supported in part by NSF Grant MCS 76-04661. 
COROllary 2. If $X$ is a compact subset of $S$ and if none of the complementary components of $X$ extend over more than a hemisphere of $S$, then $0 \in \hat{X}$.

Proof. If $H$ is an open hemisphere of $S$, then $0 \in(S \backslash H)^{\wedge}$.

COROLlaRY 3. If $T$ is a compact totally disconnected subset of $S$, then $(S \backslash T)^{\wedge}=\bar{B} \backslash T$.

Proof. Let $x \in B$. Choose $\varepsilon>0$ so that for any spherical cap

$$
\Delta=\{\eta \in S:|\eta-z|<\varepsilon\}
$$

with $z \in S, x \in(S \backslash \Delta)^{\wedge}$. Cover $T$ by such sets $\Delta_{1}, \ldots, \Delta_{N}$. Since $T$ is totally disconnected, there are disjoint open subsets of $S$, say $U_{1}, \ldots, U_{N}$, with $U_{j} \subseteq \Delta_{j}$ for each $j$ and with $U_{j}$ still covering $T$. Then $X=S \backslash \cup U_{j}$ is a compact subset of $S \backslash T$, and from the theorem $x \in \hat{X}$. Thus $x \in(S \backslash T)$.

The main result needed to prove the theorem is the following lemma, which makes essential use of Alexander's result.

LEMMA. If $X$ is a compact subset of $S$, then $\partial \hat{X} \cap B \subseteq\left[\partial_{S} X\right]^{\wedge}$.

Proof. If the lemma is false, then there is a compact set $X \subseteq S$, a point $x \in \partial \hat{X} \cap B$, and a polynomial $p$ with

$$
\begin{gathered}
p(x)=1+\delta, \quad \delta>0 \\
|p| \leqslant 1 \quad \text { on } \partial_{S} X .
\end{gathered}
$$

We will show that this leads to a contradiction.

Let $K=\{z \in S|| p(z) \mid \leqslant 1+\delta / 2\}$, and note that $\partial_{S} X \subseteq K$. Let $V$ be the component of $B \backslash \hat{K}$ which contains $x$. From the proof of Theorem 3 in [1], it follows that there is a unique component $U$ of $S \backslash K$ for which $U \cap \bar{V} \neq \varnothing$. Since $U$ is connected and $U \subseteq S \backslash K \subseteq S \backslash \partial_{S} X$, either $U \subseteq X$ or $U \subseteq S \backslash$ $X$. We consider these two possibilities separately.

Case I. Suppose $U \subseteq X$. Let $z \in V$. Let $W=\left\{\eta \in \mathbf{C}^{n}: p(\eta)=p(z)\right\}$. By the maximum principle for analytic varieties, we have that for all polynomials $q,|q(z)| \leqslant \max _{W \cap \partial V}|q|$. But $\partial V \subseteq \bar{U} \cup\{\eta \in B:|p(\eta)|=1+\delta / 2\}$, whence $|q(z)| \leqslant \max _{\bar{U}}|q| \leqslant \max _{X}|q|$. Thus $x \in V \subseteq \hat{X}$; but this contradicts $x \in \partial \hat{X}$.

Case II. Suppose $U \subseteq S \backslash X$. Let $L=\hat{X} \cap \bar{V}$, and let $m=\max _{L}|p|$. Since $x \in L, m \geqslant 1+\delta$. Choose $y \in L$ such that $|p(y)|=m$, and let

$$
T=\{z \in L: p(z)=p(y)\} .
$$

Observe that $T \subseteq \hat{X} \cap V$, since $|p| \geqslant 1+\delta$ on $T$ while $\partial V \subseteq \bar{U} \cup\{z \in B$ : $|p(z)|=1+\delta / 2\}$ and $\hat{X} \cap \bar{U} \subseteq \partial_{S} X$ where $|p| \leqslant 1$. But then $T$ is a local peak set for $P(\hat{X})$, hence a peak set for $P(\hat{X})$, which contradicts $T \subseteq V \subseteq B$.

Proof of THEOREM. Observe that it suffices to prove the theorem for the case $N<\infty$, since the infinite case can be obtained from the finite one by taking a decreasing intersection. It is trivial that $\left(S \backslash \cup U_{j}\right)^{\wedge} \subseteq \cap\left(S \backslash U_{j}\right)^{\wedge}$. To prove the reverse inclusion it is evidently sufficient to show that $x$ $\in \partial\left[\cap\left(S \backslash U_{j}\right)^{\wedge}\right] \cap B$ implies $x \in\left(S \backslash \cup U_{j}\right)^{\wedge}$. Since $N<\infty$, there is some $j$ 
for which $x \in \partial\left[\left(S \backslash U_{j}\right)^{\wedge}\right] \cap B$, so by the lemma $x \in\left[\partial_{S}\left(S \backslash U_{j}\right)\right]^{\wedge}$. Since the $U_{j}$ are disjoint, $\partial_{S}\left(S \backslash U_{j}\right) \subseteq S \backslash \cup U_{j}$, so $x \in\left(S \backslash \cup U_{j}\right)^{\wedge}$ as desired.

\section{REFERENCES}

1. H. Alexander, A note on polynomial hulls, Proc. Amer. Math. Soc. 33 (1972), 389-391.

Department of Mathematics, Lehigh University, Bethlehem, Pennsylvania 18015 